\title{
Subband Decomposition Techniques for Adaptive Channel Equalisation
}

\author{
Hafizal Mohamad ${ }^{1}$, Stephan Weiss ${ }^{2}$, Nor Azhar Mohd Arif ${ }^{1}$ and Mohamad Yusoff Alias ${ }^{1}$ \\ ${ }^{1}$ Faculty of Engineering, Multimedia University, Cyberjaya, Selangor, Malaysia \\ ${ }^{2}$ School of Electronics \& Computer Science, University of Southampton, UK \\ \{hafizal, norazhar, yusoff\}@mmu.edu.my, sw1@ecs.soton.ac.uk
}

\begin{abstract}
In this contribution, the convergence behaviour of the adaptive linear equaliser based on subband decomposition technique is investigated. Two different subband-based linear equalisers are employed, with the aim of improving the equaliser's convergence performance. Simulation results over three channel models having different spectral characteristic are presented. Computer simulations indicate that subband-based equalisers outperform the conventional fullband linear equaliser when channel exhibit severe spectral dynamic. Convergence rate of subband equalisers are governed by the slowest subband, whereby different convergence behaviour in each individual subband is observed. Finally, the complexity of fullband and subband equalisers is discussed.
\end{abstract}

\section{INTRODUCTION}

In digital communication systems, each symbol transmitted over a time-dispersive channel will be blurred over a time interval larger than a symbol period. Linear channel distortions caused by multi-path propagation and limited bandwidth lead to inter-symbol interference (ISI) at the receiver, which in turn results in a high bit error rate in the detection. ISI is one of the major obstacles to reliable high-speed data transmission over wireless channels of limited bandwidth [1].

Linear equaliser (LE) has been widely employed to mitigate the effect of ISI for its simple implementation. However, there are some limitation imposed to LE, for instance, poor performance of LE was reported when channel exhibits spectral dynamics [1]. A general channel and equaliser block diagram is depicted in Fig. 1, where the equaliser has the task to undo distortion exhibited by the channel. The equaliser $\mathbf{w}[n]$ lies in cascade between the propagation channel $c[n]$ and the decision circuit, where $x[n]$ and $y[n]$ are the equaliser's input and output signals, respectively. The transmitted symbol is denoted by $u[n]$, while the noise corrupting the received signal $x[n]$ is represented by $v[n]$.

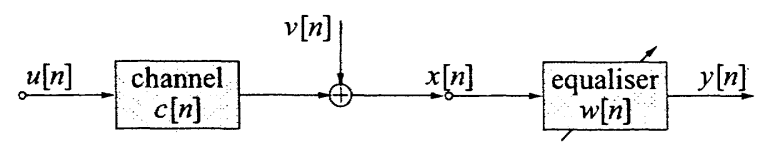

Fig. 1. General channel and equaliser block diagram.

Least mean square (LMS) algorithm is popular in numerous adaptive filtering applications, including adaptive equalisation, due to its simplicity in implementation and its robustness characteristic. It is also known that the convergence speed of LMS algorithm is inversely proportional to the eigenvalue spread of its input signal [2]. The conventional linear equaliser may suffer from slow convergence due to strong spectral dynamics at the input of the equaliser [3]. Alternatively, by using recursive least square (RLS) algorithms, faster convergence speed can be obtained at the cost of increasing the computational complexity of order $\mathcal{O}\left(L^{2}\right)$, where $L$ denotes the filter length [4].

Motivated by the advantage of prewhitening effect of the filter banks and parallelisation properties [5], [6], notable success of subband decomposition method in adaptive filtering applications e.g echo cancellation has been reported in [7]. Therefore, in this paper, we investigate the performance of subband-based linear equaliser with the aim of increasing the convergence speed while maintaining the low complexity of the LMS algorithm of order $\mathcal{O}(L)$. Two different subband equaliser structures are employed to improve the equaliser's performance in terms of mean square error (MSE) convergence behaviour as compared to the conventional linear equaliser benchmark.

The remaining of this paper is organised as follows. In Section II, we briefly describe the channel characteristics, eigenvalue spread and their relation to the convergence of LMS-type algorithm. Then, we introduce various fullband and subband-based linear equaliser structures as well as discussing their computational complexity in Section III. Finally, in Section IV, we present some simulation results to demonstrate the performance of the subband decomposition approach.

\section{CHANNEL CHARACTERISTICS}

In this section, we introduce three discrete-time channels that will be used in our simulations. The channel models are listed in Tab. I. The three channels exhibit varying degrees of spectral dynamic as shown in Fig. 2, whereby the minimum magnitude of Channel 1,2 and 3 are $-60 \mathrm{~dB},-25 \mathrm{~dB}$ and $-8 \mathrm{~dB}$, respectively. The channels represents different spectral dynamic with Channel 1 displays an example of mild channel that exhibits less spectral dynamic. The worst spectral dynamic amongst the three channels is represented by Channel 3, while Channel 2 provides the medium spectral dynamic in comparison to Channel 1 and Channel 3.

An illustration of a generalised discrete Fourier transform (GDFT) modulated filter banks having $\mathrm{K}=4$ number of subbands are indicated by the dotted lines in Fig. 2. We are 


\begin{tabular}{ll} 
Channel Label & Channel Impulse Response (CIR) \\
\hline \hline Channel 1 & $0.182+0.269 z^{-1}+0.888 z^{-2}$ \\
& $+0.269 z^{-3}+0.182 z^{-4}$ \\
\hline Channel 2 & $\begin{array}{l}0.292+0.360 z^{-1}+0.756 z^{-2} \\
+0.360 z^{-3}+0.292 z^{-4}\end{array}$ \\
& $\begin{array}{l}0.227+0.460 z^{-1}+0.688 z^{-2} \\
\text { Channel 3 }\end{array}$ \\
& TABLE I
\end{tabular}

STATIC DISPERSIVE CHANNEL IMPULSE RESPONSES

now sub-dividing the whole channel models spectrum into 4 smaller frequency bands (so-called "subbands"), which in turn will reduce the channel output eigenvalue spread within each subband. The eigenvalue spread can be approximated by the ratio between the maximum and minimum value of the channel power spectral dynamic. Note that the deep channel spectrum is located in the $2^{\text {nd }}$ and $3^{\text {rd }}$ subbands covering the frequency interval $\Omega / \pi=[0.5 ; 1.0]$ and $\Omega / \pi=[1.0 ; 1.5]$, respectively. On the other hand, observe that within $1^{\text {st }}$ and $4^{\text {th }}$ subbands represent channel spectrums that exhibit less spectral dynamic, viz. less eigenvalue spread.

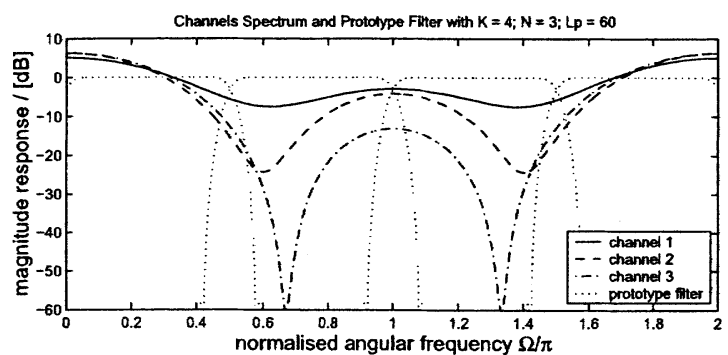

Fig. 2. Channel spectral characteristic for three channels listed in Tab. I. An example of GDFT modulated filter banks with $K=4$ number of subbands is also shown by the dotted lines indicating the band edges.

\section{EQUALISER STRUCTURES}

A general schematic of a linear equaliser is depicted in Fig. 3, whereby $x[n]$ and $y[n]$ denote the equaliser's input (received signal) and equaliser's output (equalised signal), respectively. In the following, we will derive error formulation using LMS-type algorithm for minimum mean square error (MMSE) adaptation. Let the input signal to the equaliser as $\mathbf{x}(n)$ where

$\mathbf{x}(n)=\left[\begin{array}{llll}x(n) & x(n-1) & \cdots & x\left(n-L_{\text {fullband }}-1\right)\end{array}\right]^{T}$

and $L_{\text {fullband }}$ denotes the length of fullband equaliser.

The error signal $e(n)$ is defined as the difference between the training sequence $d(n)$ and the output through the equaliser $y(n)$,

$$
e(n)=d(n)-y(n)=d(n)-\mathbf{w}(n)^{H} \mathbf{x}(n)
$$

By applying a normalised LMS (NLMS) algorithm, the weight vector at time $n+1$ is updated by

$$
\mathbf{w}(n+1)=\mathbf{w}(n)+\mu \frac{\mathbf{x}(n) e^{*}(n)}{\mathbf{x}(n)^{H} \mathbf{x}(n)+\alpha}
$$

where $\mu$ is the step size, while $\alpha$ is a constant value.

It is known that LMS-type algorithm suffers from slow convergence speed when the input signal to the adaptive equaliser $\mathbf{x}(n)$ is correlated [2]. As mentioned in Section II, the convergence rate very much depends on the eigenvalue spread of the equaliser input signal. To circumvent this problem, we employ two subband structures in order to increase the equaliser's convergence rate [5], [6], [7] that decompose the equaliser input $\mathrm{x}(n)$ by using filter banks implementation.

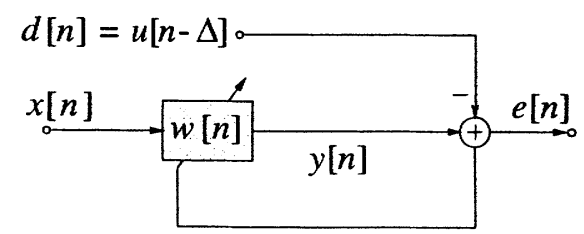

Fig. 3. Block diagram of linear fullband adaptive equaliser.

\section{A. Subband Equaliser Structures}

A first structure of subband equaliser, called Structure $I$, is shown Fig. 4, whereby $\mathbf{H}$ and $\mathbf{G}$ denote analysis and synthesis filter bank blocks including decimation and expansion as given in Fig. 5. The filters in both analysis and synthesis bank are bandpass filters, which, together with the decimation process yield a prewhitening of the subband signals compared to the input.

The system block $\mathbf{W}$ is a diagonal polynomial matrices representing filters for weight updating within each of the $K$ subbands. Adaptive filtering can be operated in each band independently, which lends itself to a parallel implementation. However, subband structures introduce aliasing that limits the algorithm performance. Therefore, oversampled filter banks (OSFB) with an oversampling ratio $K / N>1$ are preferred here [5].

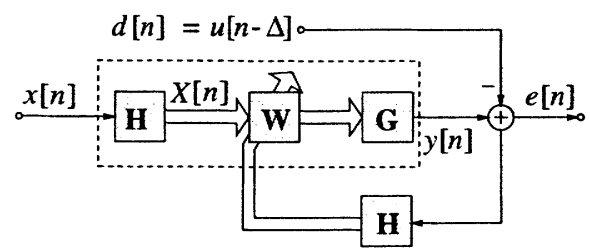

Fig. 4. Block diagram of subband Structure $I$ adaptive equaliser.

Let the subband decomposition by analysis filter bank $\mathbf{H}$ divide the sequence at the equaliser input, $\mathbf{x}(n)$, into $K$ subband sequences, $\mathbf{x}^{(1)}(n), \cdots, \mathbf{x}^{(K)}(n)$, where the superscript $(k)$ denotes the data component at the $k$ th subband. We define

$$
\mathbf{X}(n)=\left[\begin{array}{llll}
\left(\mathbf{x}^{(1)}(n)\right) & \left(\mathbf{x}^{(2)}(n)\right) & \cdots & \left(\mathbf{x}^{(K)}(n)\right)
\end{array}\right]
$$




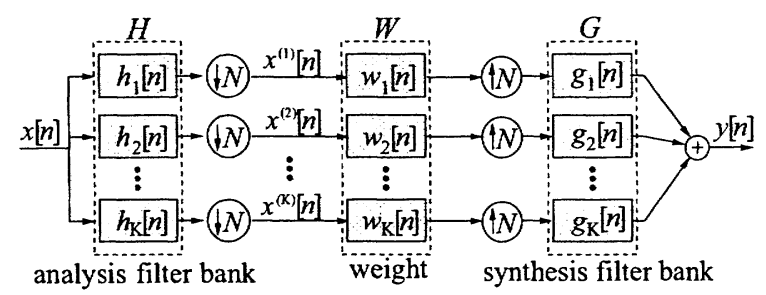

Fig. 5. Subband decomposition using $K$ number of subband decimated by a factor of $N$ with analysis filters $\mathbf{H}$ and synthesis filters $\mathbf{G}$. An independent filter weight update $\mathbf{W}$ is also shown.

as the data vector for the subband equaliser with

$$
\mathbf{x}^{(k)}(n)=\left[\begin{array}{lll}
x^{(k)}(n) & \cdots & x^{(k)}\left(n-L_{\text {subband }}-1\right)
\end{array}\right]
$$

where $L_{\text {subband }}$ denotes the length of subband equaliser. Therefore, the error signal within each subband becomes

$$
e^{(k)}(n)=d(n)-y(n)=d(n)-\mathbf{w}^{(k)}(n)^{H} \mathbf{x}^{(k)}(n)
$$

The error is evaluated based on reconstructed $y(n)$ by synthesis filter bank $\mathbf{G}$, and is projected back into the subband domain to update the filters in the weight vector $\mathbf{W}^{(k)}$, defined as

$$
\mathbf{W}(n)=\left[\begin{array}{llll}
\left(\mathbf{w}^{(1)}(n)\right) & \left(\mathbf{w}^{(2)}(n)\right) & \cdots & \left(\mathbf{w}^{(K)}(n)\right)
\end{array}\right]
$$

The system block $\mathbf{W}$ is a diagonal polynomial matrices representing filters for weight updating within each of the $K$ subbands. Adaptive filtering can be operated in each band independently, which lends itself to a parallel implementation. The adaptive subband-based can implement a normalized LMS (NLMS) algorithm, where the adaptation of the weight vector are carried out independently in each subband and is updated by

$$
\mathbf{w}^{(k)}(n+1)=\mathbf{w}^{(k)}(n)+\mu \frac{\mathbf{x}^{(k)}(n) e^{(k) *}(n)}{\mathbf{x}^{(k)}(n)^{H} \mathbf{x}^{(k)}(n)+\alpha}
$$

As an alternative subband implementation and for comparison purpose, we will modify the subband Structure $I$ and forming a new subband equaliser structure. The new subband structure will evaluate error and update weight vector coefficients in subband domain. The schematic of subband Structure II is shown in Fig. 6.

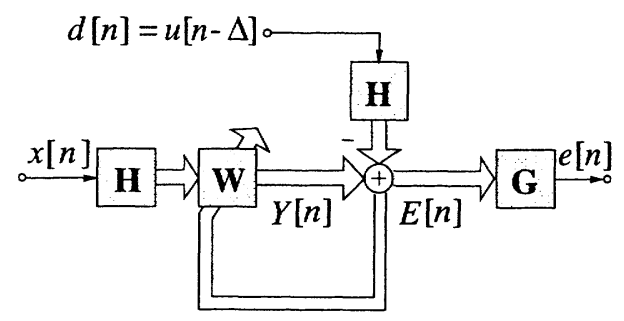

Fig. 6. Block diagram of subband Structure $I I$ adaptive equaliser.
For the derivation, we will follow the same definition that was presented for Structure I. The error formulation for Structure $I I$ is define as,

$$
E(n)=D(n)-Y(n)=D(n)-\operatorname{diag}\left\{\mathbf{W}^{\mathrm{H}}(n) \mathbf{X}(n)\right\}
$$

where the capital letter denotes the operations in subband domain and the definition is according to those in Eqn. 6.

The estimates of the weight vector is given by

$$
\mathbf{W}(n+1)=\mathbf{W}(n)+\frac{\mu}{\mathbf{P}} \mathbf{X}(n) \operatorname{diag}\left\{E^{*}(n)\right\}
$$

where $\mathbf{P}=\operatorname{diag}\left[P^{(k)}, k=1,2, \cdots ; K\right]$ is a diagonal matrix with the signal power at the $k$ th subband $P^{(k)}=E\left[\left(\mathbf{x}^{(k)}\right)^{H} \mathbf{x}^{(k)}+\alpha\right]$ as its $k$ th diagonal element.

From both derivation for subband Structure I and Structure II for LMS-type algorithm, it is expected for subband to converge faster then the fullband counterpart, as the equaliser input $\mathbf{x}(n)$ will be decorrelated by the prewhitening effect of the filter banks. Furthermore, the eigenvalue spread of the input to the equaliser will be reduced in each sub-divided subband implementation [8], as evident in Section II.

\section{B. Computational Complexity}

An impulse response in the decimated subband domain can be modelled with less equaliser coefficients than required in the fullband case due to the increased sampling period, thereby achieving. In general, the length of subband equaliser coefficients, $L_{\text {subband }}$, is given by [7],

$$
L_{\text {subband }}=\frac{L_{\text {fullband }}+2 \cdot L_{p}}{N}
$$

where $N$ is the decimation factor and $L_{\text {fullband }}$ is the equivalent fullband equaliser length. Note that $L_{p}$ is the length of a filter used in the filter bank implementation, which is often refereed to as prototype filter. In (11), subband equaliser reduces the necessary fullband equaliser length by a factor of $N$, whereby a moderate overhead of $L_{p}$ has to be taken into account as in the subband domain potentially fractional delays have to be modelled.

The complexity of the fullband equaliser expressed in terms of the number of multiply-accumulate (MAC) when using an NLMS algorithm for updating is approximately given by

$$
C_{\text {fullband }}=4 \cdot 2 \cdot L_{\text {fullband }}=8 \cdot L_{\text {fullband }} \quad[\mathrm{MAC}] \text {, }
$$

where $L_{\text {fullband }}$ denotes the number of filter taps in the fullband equaliser. As the complex input signal is utilised throughout this treatise, the equaliser taps are also complex valued. Since each complex multiplication is equivalent to 4 real multiplication, the factor of 4 in (12) accounts for the required complex valued arithmetic.

In the context of subband equaliser, the complexity of the filter banks has to be taken in consideration. In a fast implementation, one GDFT analysis or synthesis filter bank operation requires

$$
C_{\text {filter bank }}=\frac{1}{N} \cdot\left(2 \cdot L_{p}+4 \cdot K \cdot \log _{2}(K)+8 \cdot K\right)
$$


number of MAC operations per fullband sampling period [9], where $N, K$ and $L_{p}$ denote the decimation factor, the number of subbands and prototype filter length, respectively. Thus the complexity of both subband-based equaliser Structures I and Structure II utilising 3 filter banks and using the NLMS adaptive algorithm is given by

$$
C_{\text {subband }}=\frac{K}{N} \cdot 8 \cdot L_{\text {subband }}+3 \cdot C_{\text {filter bank }} \quad[\mathrm{MAC}] \text {. }
$$

\section{SIMULATION RESULTS}

In this section, simulation results and discussion concerning the performance of the conventional fullband and the proposed subband equalisers introduced in Sec. III are presented. For computer simulations, we consider a quadrature amplitude modulation (QAM) system having a 64QAM constellation transmitted over two different channel models displayed in Fig. 2. An NLMS algorithm with a step size of $\mu=0.4$ is utilised for adaptation of the fullband and subband equalisers. The signal to noise ratio (SNR) is set at $\mathrm{SNR}=30 \mathrm{~dB}$ for all simulations. The performance of both fullband and subband equalisers is measured in terms their mean squared error (MSE) convergence behaviour, whereby both the transient and steady state behaviour are of interest.

In the following subsections, we investigate the MSE performance comparison for fullband and subband equalisers utilising $\mathrm{K}=4$ subbands. Further, the individual $\left(1^{s t}, 2^{\text {nd }}, 3^{r d}\right.$ and $4^{\text {th }}$ ) subbands convergence speed is analysed. The computational complexity for different system designs employing different number of subbands is also investigated.

\section{A. MSE Performance Comparison of Fullband and Subband Equalisers}

The MSE convergence performance comparison of the fullband and subband equalisers over two different channels is depicted in Fig. 7. The MSE convergence curves were averaged over an ensemble of 20 runs.

For subband equalisers, the OFSBs splits the fullband signal into $K=4$ number of subbands signals decimated by a factor of $N=3$ with the prototype filter length of $L_{p}=60$. The length of fullband filter was selected to be $L_{f}=100$, while for the same modelling capability, the corresponding subband filter length of $L_{s}=53$ was chosen.

Observe that for Channel 1 that has a mild spectrum dynamic, the MSE convergence performance of fullband is comparable to subband equalisers performance as shown in Fig. 7 (top). However, for Channel 2, a slow convergence rate of fullband equaliser is noticeable in in Fig. 7 (middle).

For a severe spectrum dynamic Channel 3, the convergence speed of the subband equalisers considerably outperform the fullband equaliser as evidence in Fig. 7 (bottom). The superiority of the subband equaliser performance can be attributed to the reduction in eigenvalue spread via sub-dividing severe spectrum dynamic channel into a smaller frequency bins.

\section{B. MSE Convergence of Individual Subbands}

The subband decomposition of the received signal, which has been transmitted over three channel models, enables a reduction in eigenvalue spread values with each subband. The eigenvalue spread can be approximated by the ratio of the maximum and the minimum spectral dynamic in each subbands. Due to different eigenvalue spread for within each $\mathrm{K}=4$ subbands, the learning characteristic of an individual subband is different. The MSE convergence behaviour with respect to the individual subband's eigenvalue spread for subband-based equaliser is shown in Fig. 8.
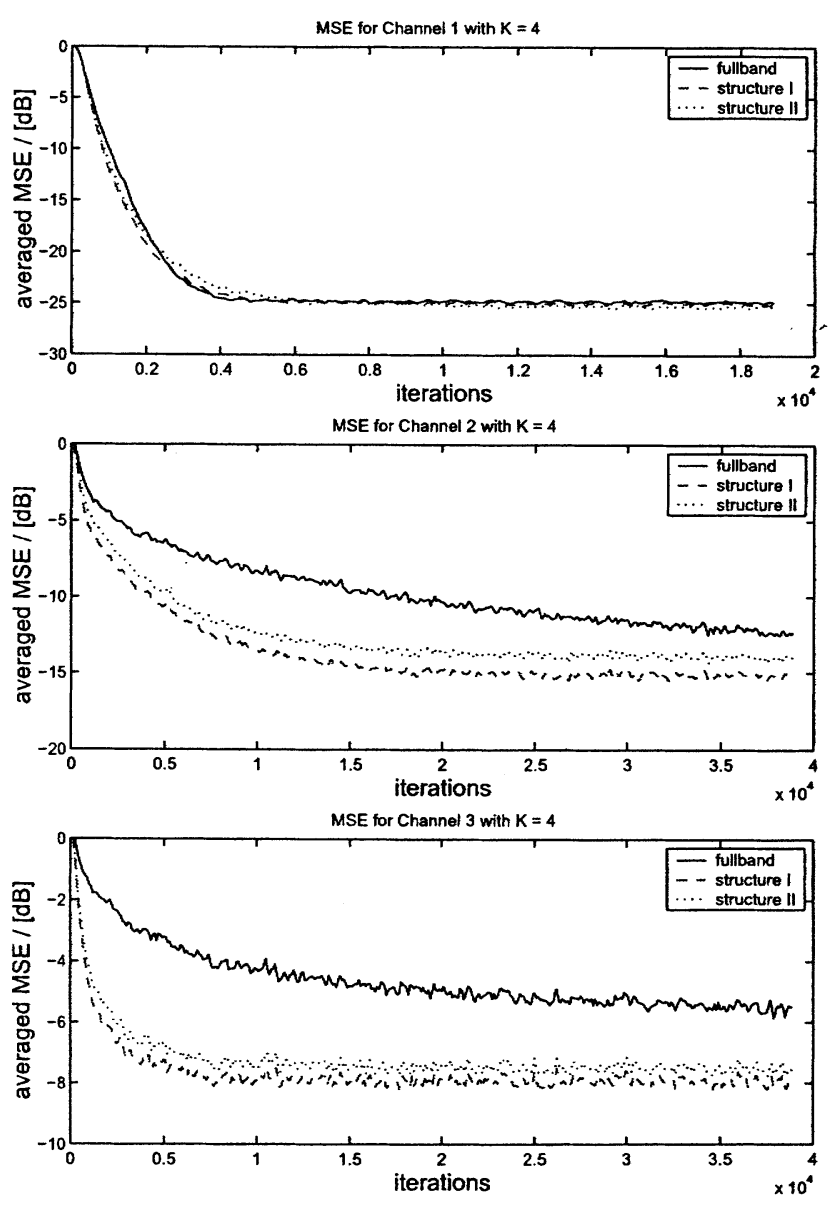

Fig. 7. MSE performance of the fullband, Structure $I$ and Structure $I I$ for (top) Channel 1, (middle) Channel 2, and (bottom) Channel 3, whereby $\mathbf{K}=$ 4 subbands were employed.

Due to different eigenvalue spread for each $\mathrm{K}=4$ subbands, the learning characteristic of an individual channel is different. The simulation results for subband Structure $I$ are shown in Fig. 8. It is indicative that the overall MSE performance for the simulated channels is influence by the convergence of the slowest subbands. Note that for all three channels, the $2^{\text {nd }}$ and $3^{r d}$ subbands that encompasses high eigenvalue spread limits the overall achievable MSE convergence of the equaliser. 

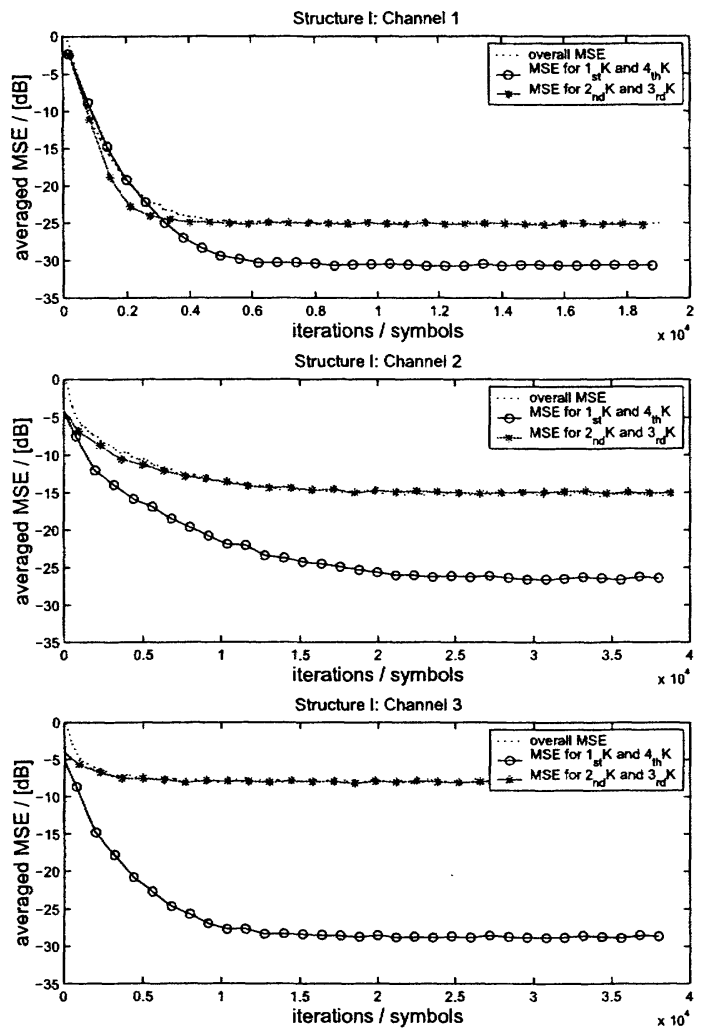

Fig. 8. Illustration of individual subband MSE convergence behaviour of the $\mathrm{K}=4$ subband Structure I for (top) Channel I, (middle) Channel 2, and (bottom) Channel 3.

\section{Computational Complexity}

In Section III we have noted that the subband equaliser length $L_{\text {subband }}$ is shorter than the fullband equaliser length $L_{\text {fullband }}$ due to operating in the decimated domain. This reduction of $L_{\text {subband }}$ with respect to $L_{\text {fullband }}$ obeys (11). The required equaliser length of fullband and subband systems is shown in Fig. 9, where the legend indicates different implementations varying the number of subbands $K$. Observe that as $K$ increases, the required subband equaliser length $L_{\text {subband }}$ decreases. For example, if a fullband equaliser requires $L_{\text {fullband }}=300$ number of taps, the corresponding subband equaliser having $K=4$ subbands will need $L_{\text {subband }}=140$ filter coefficients per subband, whereas the subband equaliser associated with $K=32$ requires $L_{\text {subband }}=$ 65 filter taps for achieving the similar modelling capabilities as the fullband system.

Consider a fullband equaliser and a subband equaliser in $K=4$ subbands with $N=3$ and $L_{p}=60$. Assuming $L_{\text {fullband }}=150$, we obtain $L_{\text {subband }}=90$ according to (11). The complexity of the fullband equaliser as given in (12) is $C_{\text {fullband }}=8 \cdot L_{\text {fullband }}=8 \cdot 150=1200$ MACs. For the subband realisation, the complexity of the filter bank implementation has to be taken into consideration and is given in (13) as $C_{\text {filter bank }}=\frac{1}{3} \cdot\left(2(60)+4(4) \log _{2} 4+8(4)\right) \approx$

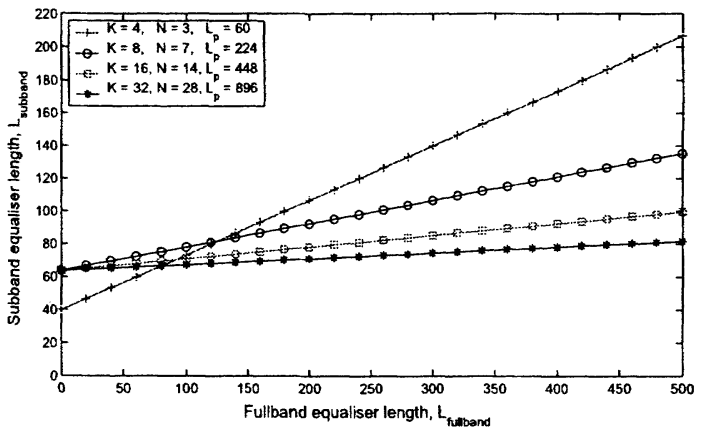

Fig. 9. Equivalence of fullband equaliser length $L_{\text {fullband }}$ and subband equaliser length $L_{\text {subband }}$ calculated according to (11).

61 MACs. Therefore, the complexity of the subband equaliser based on (14) is $C_{\text {subband }}=\frac{4}{3} \cdot 8 \cdot 90+3 \cdot 61=1143$ MACs. For the above calculation, it is observed that the subband equaliser gains a reduction in computational cost in comparison to its fullband counterpart.

\section{CONCLUSIONS}

In this paper, we investigate two structures for subband adaptive equalisation. Simulations results indicate the clear advantage of using subband-based equaliser when channel exhibits spectral dynamic, while maintaining the similar performance for mild channel in comparison to the fullband equaliser benchmark. Convergence rate of subband equalisers are governed by the slowest subband having a large spectral dynamic in comparison to other subbands. In terms of computational complexity, subband structures are very useful when a lengthy equaliser response is needed to cope with a multipath channel that exhibits a large delay spread. The advantages of the subband equliser structures can be exploited for numerous potential applications such as broadband wireline communications, e.g. ADSL modems.

\section{REFERENCES}

[1] J. G. Proakis, Digital Communication, 4th Ed, Mc-Graw Hill International, 2000.

[2] B. Widrow and S. D. Stearns, Adaptive Signal Processing, Prentice Hall, Englewood Cliffs, New York, 1985.

[3] M. Rupp, "On the Learning Behaviour of Decision Feedback Equalizers," in Asilomar Conference on Signals, Systems, and Computers, Monterey, CA, October 1999.

[4] S. Haykin, Adaptive Filter Theory, Prentice Hall, Upper Saddle River, N.J., 1996.

[5] W. Kellermann, "Analysis and Design of Multirate Systems for Cancellation of Acoustical Echoes," in Proc. IEEE ICASSP, vol. 5, pp. 2570-2573, New York, 1988

[6] J. J. Shynk, "Frequency-Domain and Multirate Adaptive Filtering," IEEE Signal Processing Magazine, vol. 9, pp. 14-37, January 1992.

[7] A. Gilloire and M. Vetterli, "Adaptive Filtering in Subbands with Critical Sampling: Analysis, Experiments and Applications to Acoustic Echo Cancellation," IEEE Transactions on Signal Processing, vol. SP-40, no. 8, pp. 1862-1875, August 1992.

[8] S. S. Pradhan and V. U. Reddy, "A New Approach to Subband Adaptive Filtering," IEEE Transactions on Signal Processing, vol. 47, no. 3, pp. 655-664, March 1999.

[9] S. Weiss and R. W. Stewart, "Fast Implementation of Oversampled Modulated Filter Banks," IEE Electronics Letters, vol. 36, no. 17, pp. 1502-1503, August 2000. 